\title{
AN INEQUALITY CONCERNING POLYHEDRA ${ }^{1}$
}

\author{
LÁSZLÓ FEJES TÓTH
}

In 1897, at the mathematical competition of the Lorand Eötvös Mathematical and Physical Society, Professor L. Fejerr, at the time still a student, noted the following interesting corollary of a well known elementary geometrical theorem of Euler: ${ }^{2}$

If $R$ denote the radius of the circumscribed circle and $r$ the radius of the inscribed circle of a given triangle, then

$$
R \geqq 2 r \text {. }
$$

This is easily established, since according to the theorem of Euler mentioned above, if $d$ denotes the distance between the centers of the circumscribed and inscribed circles, then

$$
d^{2}=R^{2}-2 r R .
$$

It follows that $R^{2}-2 r R \geqq 0$, and therefore $R \geqq 2 r$. Equality holds only if the two circles are concentric, that is, if the triangle is equilateral.

The problem of generalizing the above result to space was proposed by Professor L. Fejér. A young mathematician, I. Ảdám, deported to Germany during the war-where all traces of him have been lost-found and communicated to Professor Fejér in 1943 a very simple proof of the above extremum property of the equilateral triangle. His proof, which may be immediately generalized to space, runs as follows:

If $\rho$ is the radius of the circle passing through the midpoints of the sides of the triangle, then $\rho=R / 2$, and all that need be shown is that $\rho$ is at least equal to the radius of the inscribed circle. This follows from the fact that the inscribed circle is the smallest among all circles which have common points with all three sides of the triangle. Such a circle is, namely, the circle inscribed in a homothetic triangle containing the original one.

Received by the editors June 15, 1947.

${ }^{1}$ Lecture held in the seminar of Professor L. Fejér in April 1946.

2 T. Rad6, On mathematical life in Hungary, Amer. Math. Monthly vol. 39 (1932) pp. 85-90; J. Kürschák, Matematikai versenytêtelek, Szeged, 1929. A sharper inequality than (1) is contained in a problem proposed by M. Schreier, Jber. Deutschen Math. Verein. vol. 45 (1935) p. 196. L. J. Mordell [Középiskolai Matematikai és Fizikai Lapok vol. 11 (1935) pp. 145-146, see also Amer. Math. Monthly vol. 44 (1937) p. 252 ] has proved an analogous better inequality conjectured by $P$. Erdös: If $R_{1}, R_{2}, R_{3}$ are the distances of an inner point in a triangle from the vertices and $r_{1}, r_{2}, r_{2}$ the distances from the sides then $R_{1}+R_{2}+R_{3} \geqq 2\left(r_{1}+r_{2}+r_{3}\right)$. 
Equality in (1) can occur only if the inscribed circle passes through all three midpoints of the sides, that is, if the triangle is equilateral.

In this paper we generalize (1) and the analogous inequality for tetrahedra $R \geqq 3 r$ to arbitrary convex polygons and polyhedra, respectively. Our main results are the following two theorems:

If $R_{n}$ and $r_{n}$ denote the radii of the least sphere containing, and the greatest sphere contained in, an $n$-verticed convex polyhedron, then
If $R_{n}$ and $r_{n}$ denote the radii of the least sphere containing, and the greatest sphere contained in, an $n$-faced polyhedron, then

$$
R_{n} / r_{n} \geqq 3^{1 / 2} \tan \frac{n}{n-2} \frac{\pi}{6} .
$$

Equality holds only for a regular Equality holds only for a regular tetrahedron, octahedron and icosa- tetrahedron, hexihedron and dohedron. decahedron.

Hence (2) is exact for $n=4,6$, and 12. Furthermore, the inequality (2) gives an exact asymptotic estimate for large values of $n$, that is, (2) gives the exact value of $\lim \inf n\left(R_{n}-r_{n}\right) / R_{n}$.

It is worth mentioning that the regular hexahedron and dodecahedron are not the "best" polyhedra among the 8 or 20 verticed polyhedra and similarly the regular octahedron and icosahedron are not the best ones among the 8 or 20 faced polyhedra. This shows that in certain extremum problems for polyhedra they are the trigonal faced, in others the trihedral verticed regular polyhedra which play a distinguished roll. The natural question to transfer other well known extremum properties of the regular polygons to the trihedral verticed or trigonal faced regular polyhedra has-as far as I know-not been treated in literature, except for the few cases quoted in this paper.

When Professor Fejér mentioned the above problem to me, I noticed that the extremum property in question can be related to the following two well known extremum properties:

(1) Among all triangles inscribable in a given circle the equilateral triangle has the greatest area.

(2) Among all triangles circumscribable about a given circle, the equilateral triangle has the smallest area.

In other words:

(1a) Among all circles circumscribed about triangles having the same area, the circle circumscribed about the equilateral triangle is the smallest.

(2a) Among all circles inscribed in triangles having the same area, the circle inscribed in the equilateral triangle is the largest. 
The circle circumscribed about a non-equilateral triangle is therefore greater, and the inscribed circle smaller, than the circles circumscribed about, and inscribed in, an equilateral triangle of the same area.

The following obvious generalization of the above results suggests itself: If $t_{n}$ and $T_{n}$ denote the areas of two ellipses the first of which is contained in an $n$-sided polygon of area $t$, and the second of which contains the same polygon, then

$$
t_{n} \frac{n}{\pi} \tan \frac{\pi}{n} \leqq t \leqq T_{n} \frac{n}{2 \pi} \sin \frac{2 \pi}{n} .
$$

It follows that for any two ellipses satisfying the above condition,

$$
\frac{T_{n}}{t_{n}} \geqq \cos ^{-2} \frac{\pi}{n} .
$$

Equality can occur only for an affine regular $n$-sided polygon.

Corresponding to this result, (2) shall be proved under the following more general conditions:

THEOREM. Let $V_{n}$ and $v_{n}$ denote the volumes of two ellipsoids, the first containing, the second contained in, a convex polyhedron having either $n$ vertices or $n$ faces. In both cases we have

$$
V_{n} / v_{n} \geqq 3^{3 / 2} \tan ^{3} \frac{n \pi}{(n-2) 6} .
$$

This inequality is exact for $n=4,6,12$ and gives an exact asymptotic estimate for large values of $n .^{4}$

This theorem is a direct generalization of the following known result: $:^{5}$ If a convex polyhedron with $n$ faces (or $n$ vertices) lies between

\footnotetext{
${ }^{3}$ Equality holds if the number of vertices is given for affine regular triangularfaced polyhedra, or if the number of faces is given for affine regular polyhedra with trihedral vertices.

- (3) gives the exact value of lim inf $n\left(V_{n}-v_{n}\right) / V_{n}$.

${ }^{5} \mathrm{~L}$. Fejes, Egy gömbfelïlet befedése egybevagó gömbsilvegekkel, Matematikai és Fizikai Lapok vol. 50 (1943) pp. 40-46. Other generalizations of (4) are the two following equivalent theorems [L. Fejes, Über einige Extremaleigenschaften der regulären Polyeder und des gleichseitigen Dreiecksgitters, Annali della R. Scuola Normale Superiore di Pisa (under publication). L. Fejes, Extremalis pontrendszerek a sikban, a gömbfeliuleten és a térben. Acta Sci. Math. et Nat. 23. Kolozsvár 1944]: If a polyhedron with $n$ faces having only trihedral vertices contains a sphere with unit radius then the arithmetic mean of the distances $R_{1}, R_{2}, \cdots, R_{2 n-1}$ between the
} 
two concentric spheres with radii $r_{n}$ and $R_{n}$, then

$$
R_{n} / r_{n} \geqq 3^{1 / 2} \tan \frac{n \pi}{(n-2) 6} .
$$

The theorem for polyhedra with $n$ vertices follows from the theorem for polyhedra with $n$ faces, and conversely. This is a consequence of the following lemma.

Lemma. Let $V_{1}, V_{2}, V_{3}$ denote three ellipsoids, $V_{1}$ and $V_{8}$ being polarreciprocals of each other with respect to $V_{2}$. Between the volumes ${ }^{6}$ of the ellipsoids the following inequality holds:

$$
V_{3} / V_{2} \geqq V_{2} / V_{1}
$$

Equality holds only if $V_{1}, V_{2}, V_{3}$ are concentric.

Proof. It can be supposed that $V_{2}$ is the unit sphere, with center at the origin, and that the $x, y, z$, axes are parallel to the principal axes, $2 a, 2 b, 2 c$, of $V_{1}$, respectively. Because, by the polar-reciprocity with respect to $V_{2}, V_{1}$ is carried into an ellipsoid, it follows that $V_{1}$ contains the center of $V_{2}$ and thus if the coordinates of the center of $V_{2}$ are $\xi, \eta, \zeta$, we have

$$
|\xi|<a, \quad|\eta|<b, \quad|\xi|<c .
$$

The reciprocity, applied to the tangent planes of $V_{1}$ at the end points of the axis of length $2 a$, yields two points of $V_{3}$, the distance between which is given by

$$
\frac{1}{a+\xi}+\frac{1}{a-\xi}=\frac{2 a}{a^{2}-\xi^{2}} \geqq \frac{2}{a} .
$$

Similar considerations applied to the other axes of $V_{1}$ yield three mutually perpendicular chords of $V_{3}$ whose lengths are not less than $2 / a, 2 / b, 2 / c$, respectively. A fortiori $V_{3}$ has three mutually perpendicular diameters of length $2 a^{\prime}, 2 b^{\prime}, 2 c^{\prime}$ such that

centre $O$ of the sphere and the vertices satisfies the following inequality: (a) $\left(R_{1}+R_{2}+\cdots+R_{2 n-1}\right) /(2 n-4) \geqq 3^{1 / 2} \tan (n /(n-2))(\pi / 6)$.

If a polyhedron with $n$ vertices bounded only by triangles is contained in a sphere with unit radius with center an inner point $O$ of the polyhedron then the harmonic mean of the distances $r_{1}, r_{2}, \cdots, r_{n+4}$ between $O$ and the faces satisfies the following inequality: (b) $(2 n-4) /\left(1 / r_{1}+1 / r_{2}+\cdots+1 / r_{2 n-4}\right) \leqq\left(3^{1 / 2} / 3\right)$ cot $(n /(n-2))(\pi / 6)$.

${ }^{6}$ In what follows we denote the area or volume of a spherical or solid domain by the same letter. 


$$
8 a^{\prime} b^{\prime} c^{\prime} \geqq \frac{8}{a b c}
$$

Let us replace one of the diameters of the octahedron with diameters $2 a^{\prime}, 2 b^{\prime}, 2 c^{\prime}$, for example, $2 b^{\prime}$, by the diameter $2 b^{\prime \prime}$ conjugate with respect to $V_{3}$ to the diameter $2 a^{\prime}$ lying in the plane $a^{\prime} b^{\prime}$. Similarly let us replace $2 c^{\prime}$ by the diameter $2 c^{\prime \prime}$ conjugate to the diametral plane $a^{\prime} b^{\prime \prime}$. The volume of the original octahedron $4 a^{\prime} b^{\prime} c^{\prime} / 3$ has been increased by both steps. The diameters of the octahedron thus obtained are pair by pair conjugate with respect to $V_{3}$ and thus if $\bar{a}, \bar{b}, \bar{c}$ denote the principal axes of $V_{3}$, we have

$$
\frac{4}{3} \bar{a} \bar{b} \bar{c} \geqq \frac{4}{3} a^{\prime} b^{\prime} c^{\prime} \geqq \frac{4}{3} \frac{1}{a b c} .
$$

Hence

$$
V_{1} V_{3}=\frac{4 \pi}{3} a b c \frac{4 \pi}{3} \bar{a} b \bar{c} \geqq\left(\frac{4 \pi}{3}\right)^{2}=V_{2}^{2},
$$

which proves the lemma.

Let us suppose now that the statement of the theorem concerning the number of vertices has been proved. Let $P_{n}$ denote a convex $n$-faced polyhedron, $v_{n}$ an ellipsoid contained in $P_{n}, V_{n}$ an ellipsoid containing $P_{n}$. Taking polar reciprocals with respect to $v_{n}$, there corresponds to $P_{n}$ a polyhedron with $n$ vertices contained in $v_{n}$ and containing the ellipsoid $V_{n}^{\prime}$ reciprocal to $V_{n}$. According to the lemma and our hypothesis

$$
V_{n} / v_{n} \geqq v_{n} / V_{n}^{\prime} \geqq 3^{3 / 2} \tan ^{3} \frac{n}{(n-2)} \frac{\pi}{6},
$$

which is the statement of the theorem for polyhedra with $n$ faces. In a similar manner we may obtain the theorem for $n$-vertices from the theorem for $n$ faces. ${ }^{7}$

We shall prove the statement of the theorem for polyhedra with $n$ vertices.

Analogously to our considerations concerning the plane, our theorem is the consequence of two inequalities. Let $\bar{v}_{n}$ denote the volume of any $n$-verticed convex polyhedron contained in the unit sphere. Then ${ }^{8}$

7 Another interesting consequence of our lemma is that for the $n$-hedron (or for the polyhedron with $n$ vertices) which has a minimal value of $V_{n} / v_{n}$ the respective ellipsoids are concentric. This can be seen by making use twice of polar reciprocation.

${ }^{8}$ See the second and the third paper referred to in footnote 5. 


$$
\bar{v}_{n} \leqq \frac{n-2}{6}\left(3-\cot ^{2} \frac{n}{n-2} \frac{\pi}{6}\right) \cot \frac{n}{n-2} \frac{\pi}{6} .
$$

Let $\bar{V}_{n}$ be the volume of any $n$-verticed convex polyhedron containing the unit sphere. Then ${ }^{9}$

$$
\bar{V}_{n} \geqq \frac{3^{1 / 2}(n-2)}{2}\left(3 \tan ^{2} \frac{n}{n-2} \frac{\pi}{6}-1\right) .
$$

In the proof of (6) it can be supposed that the vertices $C_{i}$ of $\bar{v}_{n}$ lie on the unit sphere $S$ with centre $O$, and that all faces of $\bar{v}_{n}$ are triangular. If $C_{i} C_{j} C_{k}$ is one of the faces, the tetrahedron $O C_{i} C_{j} C_{k}$ shall be denoted by $v_{i j k}$ and the spherical triangle $C_{i} C_{j} C_{k}$ by $f_{i j k}$. It will be shown that if the area of $f_{i j k}$ is given, $v_{i j k}$ has maximal volume if $C_{i} C_{j} C_{k}$ is equilateral.

The existence of the extremum being assured by Weierstrass' theorem, it is sufficient to show that, for instance, in the case $C_{i} C_{k} \neq C_{k} C_{j}$, the volume $v_{i j k}$ can be increased. For that purpose let us move $C_{k}$ on the Lexell circle passing through the points $C_{i}^{\prime}, C_{j}^{\prime}$ diametrically opposed to $C_{i}, C_{j}$ respectively, and through the original position of $C_{k}$. The area of $f_{i j k}$ remains unaltered and the volume of $v_{i j k}$ takes its maximal value if $C_{i} C_{k}=C_{k} C_{j}$, since the height of the tetrahedron drawn from the point $C_{k}$ is in every other case less.

This extremum property is expressed-making an elementary calculation-by the inequality

$$
v_{i j k} \leqq \frac{1}{12}\left(3-\cot ^{2} \frac{f_{i j k}+\pi}{6}\right) \cot \frac{f_{i j k}+\pi}{6} .
$$

But the function of $f_{i j k}$ which shall be denoted for the sake of brevity by $v\left(f_{i j k}\right)$ is concave from below in the interval $(0,2 \pi)$ since

$$
v^{\prime}(f)=\frac{\cos (f+\pi) / 3}{24 \sin ^{4}(f+\pi) / 6}
$$

is a monotonically decreasing function of $f$ in the interval $(0,2 \pi)$. Therefore-taking into account the fact that a convex polyhedron with triangular faces having $n$ vertices has $2 n-4$ faces-by adding the last inequalities for each face of $\bar{v}_{n}$, and by using Jensen's inequality $^{10}$ we obtain

- The analogous estimate concerning polyhedra with $n$ faces was found by M. Goldberg, The isoperimetric problem for polyhedra, Tohoku Math. J. vol. 40 (1935) pp. 226-236.

${ }^{10}$ Jensen, Sur les fonctions convexes et les inégalités entre les valeurs moyennes, Acta Math. vol. 30 (1906) pp. 175-193. 


$$
\bar{v}_{n}=\sum v_{i j k} \leqq \sum v\left(f_{i j k}\right) \leqq(2 n-4) v\left(\frac{4 \pi}{2 n-4}\right)
$$

which was to be proved.

(7) can be proved in a similar manner. For this purpose we use the following elementary extremum property. The central projection from $O$ of the spherical triangle $f_{i j k}$ with given surface on a plane $p$ which does not intersect $S$ has minimal area if $f_{i j k}$ is equilateral and $p$ is tangential to $S$ at the center of $f_{i j k}$. Accordingly, if the area of a face of $\bar{V}_{n}$ is denoted by $t_{i j k}$, and its projection on $S$ is denoted by $f_{i j k}$, we have

$$
t_{i j k} \geqq \frac{3^{3 / 2}}{4}\left(3 \cot ^{2} \frac{2 \pi-f_{i j k}}{6}-1\right) .
$$

The function $t\left(f_{i j k}\right)$ being convex from below in $(0,2 \pi)$, we have

$$
\bar{V}_{n} \geqq \frac{1}{3} \sum t_{i j k} \geqq \frac{1}{3} \sum t\left(f_{i j k}\right) \geqq \frac{2 n-4}{3} t\left(\frac{4 \pi}{2 n-4}\right) .
$$

Now it follows from (6) and (7) that if the ellipsoid with volume $v_{n}$ is contained in a polyhedron with $n$ vertices with volume $V$, and the ellipsoid with volume $V_{n}$ contains the same polyhedron, we have

$$
\begin{aligned}
v_{n} \frac{3}{4 \pi} \frac{3^{1 / 2}(n-2)}{2} & \left(3 \tan ^{2} \frac{n}{n-2} \frac{\pi}{6}-1\right) \\
& \leqq V \leqq V_{n} \frac{3}{4 \pi} \frac{n-2}{6}\left(3-\cot ^{2} \frac{n}{n-2} \frac{\pi}{6}\right) \cot \frac{n}{n-2} \frac{\pi}{6} .
\end{aligned}
$$

Thus (3) has been proved. The cases of equality can be immediately established. The meaning of the estimate for large values of $n$ shall be illustrated by an application.

As a corollary to our theorem we can prove that if $n>2$ spherical segments (of one base) with equal surfaces $f_{n}$ cover the whole surface of the sphere of unit radius, we have

$$
f_{n} \geqq 2 \pi\left(1-\frac{3^{1 / 2}}{3} \cot \frac{n}{n-2} \frac{\pi}{6}\right) .
$$

The "density" $d$ of this system of spherical segments is accordingly given by

$$
d=\frac{n f_{n}}{4 \pi} \geqq \frac{n}{2}\left(1-\frac{3^{1 / 2}}{3} \cot \frac{n}{n-2} \frac{\pi}{6}\right) .
$$


Since the right side is greater than $2 \cdot 3^{1 / 2} \pi / 9,11$ for every value of $n>2$, we have, for the density, $d$, of any system of congruent spherical segments smaller than the hemisphere covering the whole surface of the sphere,

$$
d>\frac{2 \cdot 3^{1 / 2} \pi}{9}(=1.209 \cdots)
$$

This remarkable inequality implies that of $\mathrm{R}$. Kershner ${ }^{12}$ which expresses the fact that the density of any system of congruent circles covering the plane is not less than $2 \cdot 3^{1 / 2} \pi / 9$.

For $n=3$, (8) expresses the simple fact that the surface of the sphere cannot be covered by three spherical segments which are smaller than the hemisphere. Therefore it is sufficient to restrict ourselves to $n \geqq 4$. Since the spherical segments cover the sphere of unit radius, the $n$-hedron $P_{n}$ determined by the planes of the bases of the spherical segments is contained in $S$. Further the heights of the spherical segments being $f_{n} / 2 \pi, P_{n}$ contains the sphere with radius $1-f_{n} / 2 \pi$. Thus according to our theorem

$$
1 /\left(1-f_{n} / 2 \pi\right) \geqq 3^{1 / 2} \tan \frac{n}{n-2} \frac{\pi}{6}
$$

which is equivalent to (8).

An analogous result holds for the density $d$ of an arbitrary system consisting of $n>2$ congruent segments of the unit sphere having no common inner points. ${ }^{13}$ In this case we have

$$
d \geqq \frac{n}{2}\left(1-\frac{1}{2} \sin ^{-1} \frac{n}{n-2} \frac{\pi}{6}\right)>\frac{3^{1 / 2} \pi}{6}(=0.907 \cdots) .
$$

BUdAPEST, Hungary

11 See the third paper in footnote 5.

${ }^{12} \mathrm{R}$. Kershner, The number of circles covering a set, Amer. J. Math. vol. 61 (1939) pp. 665-671.

${ }^{13}$ See L. Fejes, Über eine Abschätzung des kürzesten Abstandes zweier Punkte eines auf einer Kugelfä̈che liegenden Punksystems, Jber. Deutschen Math. Verein vol. 53 (1943) pp. 65-68, and the third paper referred to in footnote 5. 